\title{
Effect of oral L-citrulline on brachial and aortic blood pressure defined by resting status: evidence from randomized controlled trials
}

\author{
Huan-Huan Yang ${ }^{1}$, Xin-Li Li ${ }^{1}$, Wei-Guo Zhang ${ }^{2}$, Arturo Figueroa ${ }^{3}$, Li-Hua Chen ${ }^{1 *}$ and Li-Qiang Qin ${ }^{1 *}$
}

\begin{abstract}
Background: Experimental evidence indicates that oral L-citrulline (L-Cit) may reduce resting blood pressure (BP) as well as BP responses to exercise and cold exposure (non-resting). However, results from human intervention trials are inconsistent. This study aims to summarize the clinical evidence regarding the effects of L-Cit supplementation on brachial systolic blood pressure (SBP), brachial diastolic blood pressure (DBP), in addition to aortic SBP and aortic DBP at rest and non-resting conditions.

Methods: Multiple databases including PubMed, Embase, Cochrane library, Web of Science, and Clinical Trials were searched systematically. Randomized controlled trials of human participants were quantitatively meta-analyzed.

Results: Fourteen trials contained in eight studies were available for quantitative syntheses for brachial BP. Results showed that L-Cit supplementation significantly reduced both brachial SBP $(-4.490 \mathrm{mmHg}, 95 \% \mathrm{Cl}:-7.332$ to -1.648 , $P=0.002)$ and brachial DBP $(-3.629 \mathrm{mmHg}, 95 \% \mathrm{Cl}:-5.825$ to $-1.434, P=0.001)$. Nine of the trials were meta-analyzed for aortic BP which showed that L-Cit intervention significantly reduced aortic SBP $(-6.763 \mathrm{mmHg}, 95 \% \mathrm{Cl}:-10.991$ to $-2.534, P=0.002)$, but not aortic DBP $(-3.396 \mathrm{mmHg}, 95 \% \mathrm{Cl}:-7.418$ to $0.627, P=0.098)$. The observed reducing effects of $\mathrm{L}$-Cit appeared stronger for non-resting than for resting brachial SBP ( $P$ for difference $=0.044$ ).

Conclusion: L-Cit supplementation significantly decreased non-resting brachial and aortic SBP. Brachial DBP was significantly lowered by L-Cit regardless of resting status. Given the relatively small number of available trials in the stratified analyses and the potential limitations of these trials, the present findings should be interpreted cautiously and need to be confirmed in future well-designed trials with a larger sample size.
\end{abstract}

Keywords: Aortic, Blood pressure, Brachial, L-citrulline, Resting status

\section{Background}

Globally, approximately $20-50 \%$ of adults are affected by hypertension, a major powerful predictor of cardiovascular disease (CVD) and premature death [1]. Reduction of blood pressure (BP) is clinically crucial for reducing risk of CVD, and even a modest reduction can still confer cardiovascular benefits [2]. However, it is estimated that nearly $50 \%$ of those treated for hypertension have uncontrolled resting BP [3].

\footnotetext{
* Correspondence: chenlihua@suda.edu.cn; qinliqiang@suda.edu.cn 'Department of Nutrition and Food Hygiene, School of Public Health, Soochow University, Suzhou 215123, China

Full list of author information is available at the end of the article
}

Generally, brachial BP is used for the diagnosis and management of hypertension. However, brachial BP does not reflect aortic BP and cardiac load accurately [4]. It has been demonstrated that aortic BP and brachial BP have substantially different responses to BP-lowering drugs [5]. Furthermore, BP post isometric handgrip (IGH) [6], whole-body vibration training (WBVT) [7] and cold exposure [8] can be increased via sympathetic and muscle metaboreflex-mediated vasoconstriction compared with baseline BP. Adults with hypertension always have exaggerated exercise BP due to muscle metaboreflex overactivation [6]. This augmented exercise BP, which is not

(c) The Author(s). 2019 Open Access This article is distributed under the terms of the Creative Commons Attribution 4.0 International License (http://creativecommons.org/licenses/by/4.0/), which permits unrestricted use, distribution, and reproduction in any medium, provided you give appropriate credit to the original author(s) and the source, provide a link to the Creative Commons license, and indicate if changes were made. The Creative Commons Public Domain Dedication waiver (http://creativecommons.org/publicdomain/zero/1.0/) applies to the data made available in this article, unless otherwise stated. 
controlled by BP-lowering drugs, is an independent risk factor for cardiovascular events and mortality [9].

L-citrulline (L-Cit), a colorless, water-soluble amino acid, is an effective endogenous precursor of L-Arginine (L-Arg) [10] and nitric oxide (NO) [11, 12]. Oral L-Arg has been shown to lower BP [13]. Thus, it is biologically plausible that L-Cit may also have BP-lowering potential. A number of randomized controlled trials (RCTs) have evaluated the effects of L-Cit on BP in subjects with varying background characteristics, but the results are inconsistent [11, 12, 14-16].

Three meta-analyses [17-19] have investigated the effect of L-Cit supplementation on BP. Two $[17,18]$ were conducted to evaluate the effect of L-Cit on brachial BP. The present study added the analysis on aortic BP, since aortic $\mathrm{BP}$ is more relevant for cardiovascular risk and responsive to antihypertensive treatments than brachial $\mathrm{BP}[4,5]$. Although another review [19] took both resting brachial and aortic BP into account, an update is needed because eligible trials were missed $[15,16,20]$. Previous metaanalyses have not evaluated the influence of L-Cit on BP during conditions with increased sympathetic-mediated vasoconstriction such as exercise and cold exposure.

We performed this systematic review with metaanalysis to evaluate the effect of L-Cit supplementation on brachial and aortic BP, taking the differences between resting $\mathrm{BP}$ and non-resting $\mathrm{BP}$ into account.

\section{Methods}

\section{Literature search}

This systematic review was performed in accordance with the principles outlined in the Cochrane Handbook 5.1.0 [21] for systematic reviews of interventions. We searched multiple electronic databases including PubMed, Embase, Cochrane Library, Web of Science, and Clinical Trials for potentially relevant studies. Articles published from inception to May 5, 2019 were filtered, and search strategies are reported in Additional file 17: Table S1. In addition, reference lists of relevant trials were searched to identify more potentially eligible trials. Our search was restricted to studies published in English. Two researchers ( $\mathrm{H}-\mathrm{HY}$ and $\mathrm{X}-\mathrm{LL}$ ) independently performed the search and justification for eligibility, and any disagreement was resolved by consensus.

\section{Inclusion and exclusion criteria}

Studies were included if they met all of the following criteria: 1) the design was a RCT; 2) the participants were adults aged 18 years or older; 3 ) L-Cit was given as the only intervention by oral administration; 4) the intervention lasted at least for one week; and 5) sufficient data on BP at baseline and at the end of follow-up or the changes of BP in each group were reported. Conference abstracts or studies lacking a controlled group were not considered.

\section{Data extraction}

The following data were extracted from each of the included trials: the first author's name, year of publication, study location, study design, blinding status, sample size, dose of L-Cit, intervention duration, participants' characteristics including sex, age, health status and BP values.

\section{Quality evaluation}

According to RevMan (Cochrane Review Manager, version 5.3), several trial components including "random sequence generation", "allocation concealment", "blinding of participants and personnel", "blinding of outcome assessment", "incomplete outcome data", "selective reporting" and "other bias" were used to assess the quality of the included RCTs. The included trials were categorized as having 'high', 'low', or 'unclear' risk of bias according to the assessment criteria.

\section{Data synthesis}

STATA 14.0 (Stata Corp., College Station, TX) was used for data analyses. For parallel trials, the net changes of each outcome in intervention and control groups were calculated as differences between mean values before and after treatments. For crossover trials, the net changes were calculated as the difference in the posttreatment values of each group. Standard deviations (SDs) of BP net changes, if not reported, were calculated by the following formulas according to the Cochrane Handbook 16.1.3.2 [21]:

$$
\begin{aligned}
& R=\frac{S D_{\text {Experimen }}{ }^{2}+S D_{\text {Control }}{ }^{2}-S D_{\text {Change }}{ }^{2}}{2 S D_{\text {Experimen }} S D_{\text {Control }}} \\
& S D=\sqrt{S D_{\text {Experimen }^{2}}{ }^{2}+S D_{\text {Control }^{2}}{ }^{2}\left(2 R S D_{\text {Experimen }} S D_{\text {Control }}\right)}
\end{aligned}
$$

Heterogeneity was quantified by the Cochrane $Q$ in addition to the $I^{2}$ statistics. A fixed-effect meta-analysis was performed to pool the weighted mean difference (WMD) between experiment and control groups because of limited heterogeneity observed across the analyses. Stratified analyses by sex, age and BP resting status were also conducted, followed by meta-regression analyses exploring potential sources of heterogeneity. Both Begg's rank correlation and Egger's linear regression tests were conducted to assess the potential publication bias. $P<$ 0.05 was considered statistically significant.

\section{Results}

\section{Study selection}

The initial search yielded a total of 2389 records, of which 1150 independent records were screened after removing the duplicates. By reading further the abstracts, 36 relevant articles remained for full-text evaluations. Among these, 28 studies were further excluded because they did not have a control group $(n=7)$, used phytochemicals 
combined with L-Arg or watermelon $(n=3)$, investigated acute effects (intervention duration $<1$ week) $(n=8)$, had no adequate data $(n=2)$, were articles from the same study $(\mathrm{n}=3)$, were conference abstracts or letters to editors $(n=5)$. Finally, eight eligible studies $[11,12,14-16$, $20,22,23]$, including 14 independent trials, were included in the present meta-analysis. Study selection process is described in Fig. 1.

\section{Study characteristics}

Various characteristics of the included studies were summarized in Table 1. These studies were published between 2010 and 2017. Six studies [12, 14, 16, 20, 22, 23] were conducted in the US, one [15] in Mexico and one [11] in Japan. Five of the six US studies were from Florida, and we confirmed that they were independent of each other based on the article review in addition to correspondences with relevant authors. Four studies [11, 12, 15, 20] employed parallel design, others were crossover designed studies with a 2week washout period. Mean age of the participants ranged from 22 to 70 years. Healthy [11, 14, 16, 22], hypertensive $[12,20,23]$ or systolic heart failure [15] participants were recruited in these studies. Capsules provided by Kyowa Hakko Bio Co., Ltd. (Tokyo, Japan) or NOW Foods (Bloomingdale, IL) were supplemented in most studies, and LCit drinks were taken in one study [15]. Dosage of L-Cit ranged from $3 \mathrm{~g} /$ day to $11 \mathrm{~g} /$ day. Intervention durations varied considerably from one week to 16 weeks.

Results for risk-of-bias assessment of the included studies are summarized in Fig. 2. Although "randomization" was described, description of randomization method was lacking among six studies [11, 14-16, 22, 23] . The study by Balderas-Munoz et al. [15] had a high risk of bias on "blinding of participants and personnel" due to lack of placebo supplementation for the control group. And three studies $[12,16,20]$ had unclear biases on this term since they did not clarify the blinding method. There might potentially be "other bias" [12, 14, 16, 22], because an investigation of resting and non-resting BP was conducted simultaneously. Fortunately, there were no risk of bias on "allocation concealment", "blinding of outcome assessment", "incomplete outcome data", or "selective reporting". In addition, there was a suggestion of publication bias regarding the effect of L-Cit on brachial DBP by the Begg's test $(P=0.025)$, and on aortic DBP by both Begg's and Egger's tests (Begg, $P=0.029$; Egger, $P=0.015$ ). The overall estimates remained unchanged after using the "trim-and-fill" method to adjust for the bias.

\section{Meta-analysis of L-Cit supplementation on BP}

Compared to control, oral L-Cit resulted in BP changes from -14.00 to $4.00 \mathrm{mmHg}$, from -16.00 to $2.10 \mathrm{mmHg}$, from -15.00 to $3.00 \mathrm{mmHg}$, and from -15.00 to 2.00 $\mathrm{mmHg}$ in brachial SBP, brachial DBP, aortic SBP, and aortic DBP, respectively. Results of meta-analyses showed that LCit supplement significantly reduced brachial SBP, brachial DBP, aortic SBP, but not aortic DBP. The estimated overall WMDs were: $-4.49 \mathrm{mmHg}(95 \% \mathrm{CI}$ : -7.33 to -1.65$)$ for brachial SBP (Fig. 3-A), $-3.63 \mathrm{mmHg}$ (95\% CI: -5.82 to 1.43) for brachial DBP (Fig. 3-B), $-6.76 \mathrm{mmHg}$ (95\% CI: -

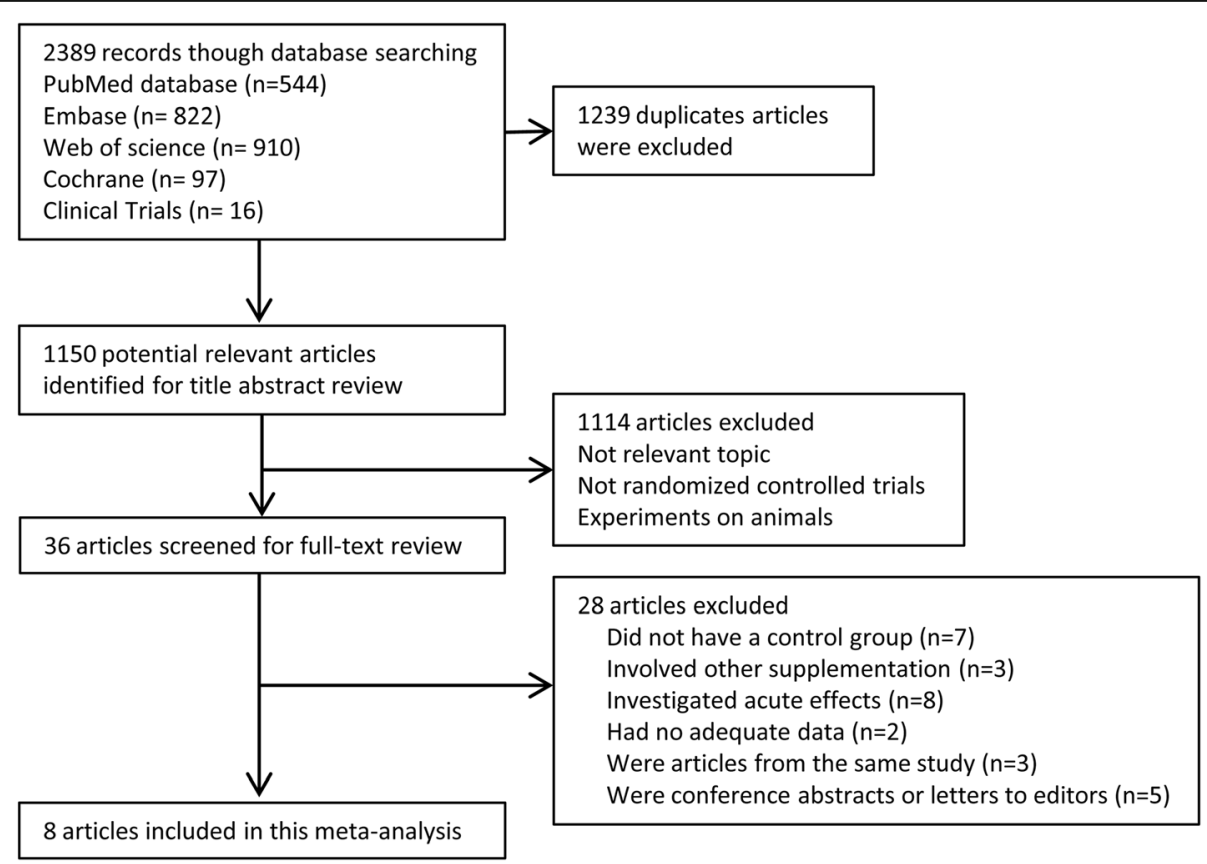

Fig. 1 Flow chart of study screening and selection 
Table 1 Baseline characteristics of the studies included in the meta-analysis

\begin{tabular}{|c|c|c|c|c|c|c|c|c|c|}
\hline \multirow[t]{2}{*}{ Author/Year } & \multirow[t]{2}{*}{ Countries } & \multirow[t]{2}{*}{ Design } & \multirow[t]{2}{*}{ Size (M/F) } & \multirow{2}{*}{$\begin{array}{l}\text { Age } \\
(\text { year } \pm S D)\end{array}$} & \multirow[t]{2}{*}{ Status } & \multirow{2}{*}{$\begin{array}{l}\text { Dose } \\
\text { (g/day) }\end{array}$} & \multirow{2}{*}{$\begin{array}{l}\text { Duration } \\
\text { (wks) }\end{array}$} & \multicolumn{2}{|c|}{ Baseline BP $(\mathrm{mm} \mathrm{Hg})$} \\
\hline & & & & & & & & L- Cit & Placebo \\
\hline Gonzales 2017 & USA & $X, D B$ & $12 / 13$ & $70 \pm 5$ & Healthy/ Hypertension & 6 & 2 & $\begin{array}{l}\text { M: } 130 \pm 13 / 65 \pm 8 \\
\text { F: } 137 \pm 16 / 77 \pm 9\end{array}$ & \\
\hline Figueroa 2016 & USA & $X, D B$ & $16 / 0$ & $24 \pm 8$ & Healthy & 6 & 2 & $123 \pm 12 / 68 \pm 8$ & $122 \pm 8 / 67 \pm 4$ \\
\hline Wong 2016 & USA & $P$ & $0 / 23$ & $58 \pm 4.8$ & Healthy/ Hypertension & 6 & 8 & $138 \pm 4 / 81 \pm 4$ & $137 \pm 4 / 80 \pm 3$ \\
\hline Wong 2015 & USA & P & $0 / 27$ & $58 \pm 3$ & Healthy/ Hypertension & 6 & 8 & $140 \pm 9 / 78 \pm 7$ & $141 \pm 2 / 80 \pm 8$ \\
\hline Sanchez-Gonzalez 2013 & USA & $x$ & $16 / 0$ & $23 \pm 12$ & Healthy & $7-11$ & 2 & $116 \pm 2 / 59 \pm 3$ & \\
\hline Balderas-Munoz 2012 & Mexico & $P, D B$ & $24 / 11$ & $67 \pm 9$ & Systolic heart failure & 3 & 16 & $113 \pm 17 / 70 \pm 12$ & $118 \pm 16 / 77 \pm 11$ \\
\hline Ochiai 2012 & Japan & $P, D B$ & $15 / 0$ & $58.3 \pm 4.4$ & Healthy & 5.6 & 1 & $136 \pm 13 / 82 \pm 7$ & $131 \pm 9 / 85 \pm 7$ \\
\hline Figueroa 2010 & USA & $X, D B$ & $17 / 0$ & $22 \pm 4.1$ & Healthy & 6 & 4 & $120 \pm 12 / 67 \pm 8$ & $121 \pm 12 / 68 \pm 8$ \\
\hline
\end{tabular}

$B P$ blood pressure; $D B$ double blinding; $F$ female; $L$ - Cit L-citrulline; $M$ male; $P$ parallel controlled trial; $S D$ standard deviation; $X$ cross-over study design

10.99 to -2.53 ) for aortic SBP (Fig. 3-C), and $-3.40 \mathrm{mmHg}$ (95\% CI: -7.42 to 0.63 ) for aortic DBP (Fig. 3-D).

\section{Stratified and meta-regression analyses}

As shown in Fig. 4, the estimated reduction of non-resting brachial SBP was $-10.17 \mathrm{mmHg}$ (95\% CI: -15.88 to -

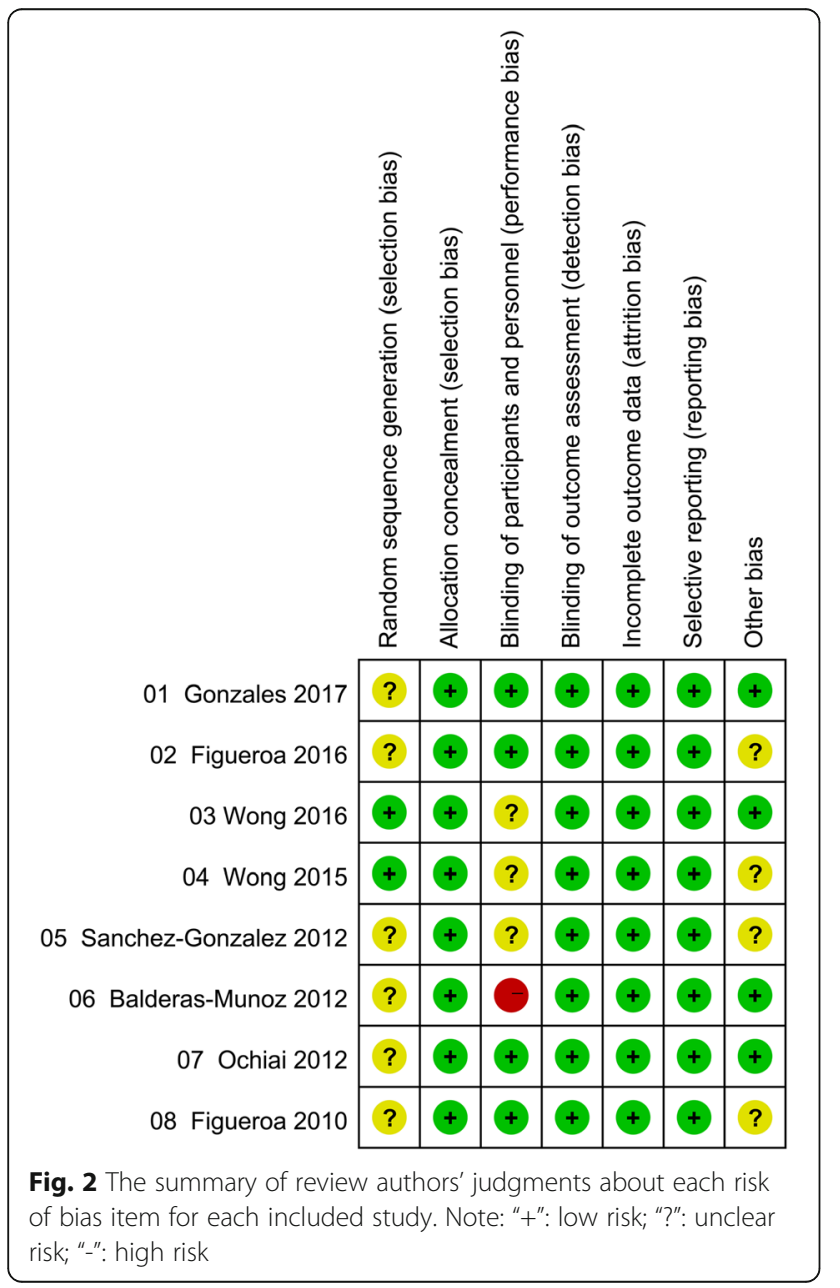

4.46), with no significant reduction of resting brachial SBP (- $2.62 \mathrm{mmHg}, 95 \% \mathrm{CI}$ : $-5.89,0.66)$. Meta-regression analyses showed that the reducing effects of L-Cit was stronger for non-resting SBP than for resting brachial SBP $(P$ for difference $=0.044$ ) (Additional file 1: Figure S1 and Additional file 2: Figure S2). Both non-resting brachial DBP $(-7.52 \mathrm{mmHg},-13.26$ to -1.79$)$ and resting brachial DBP $(-2.96 \mathrm{mmHg},-5.34$ to -0.58$)$ were significantly lowered by L-Cit supplementation ( $P$ for difference $=$ 0.175) (Additional file 3: Figure S3 and Additional file 4: Figure S4). Similar to the effect on brachial SBP, L-Cit supplementation significantly decreased non-resting aortic SBP by $10.06 \mathrm{mmHg}$ (95\% CI: -15.74 to -4.39$)$, and had no significant effect on resting aortic SBP $(-2.64 \mathrm{mmHg}$, 95\% CI: $-8.98,3.69)$. However, no significant difference was observed between them $(P$ for difference $=0.131$ ) (Additional file 5: Figure S5 and Additional file 6: Figure S6). L-Cit supplementation had no significant effect on non-resting aortic DBP $(-4.90 \mathrm{mmHg},-10.38$ to 0.59$)$ or resting aortic DBP $(-1.65 \mathrm{mmHg},-7.57$ to 4.27$)$ ( $P$ for difference $=0.456)$ (Additional file 7: Figure S7 and Additional file 8: Figure S8).

Stratified analyses found that brachial SBP was significantly reduced among men and those aged $<30$ years, but not among women or participants $>50$ years. Consistent with an overall estimated WMD, brachial DBP was significantly lowered in both men and women, as well as both age groups. No heterogeneity between groups was found in the above analysis $(P>0.05)$ (Table 2, Additional file 9: Figure S9, Additional file 10: Figure S10, Additional file 11: Figure S11, Additional file 12: Figure S12). We did not perform stratified analyses for aortic BP due to small number of studies.

In addition, a sensitivity analysis in which studies were excluded one by one was conducted. The summary WMD ranged from -5.28 (95\% CI: $-8.36,-2.21)$ to $-4.00(95 \%$ CI: $-6.91,-1.08)$ for brachial SBP, -4.03 (95\% CI: -6.30 , $-1.76)$ to -3.35 (95\% CI: $-5.58,-1.13)$ for brachial DBP, - 8.25 (95\% CI: $-12.78,-3.71)$ to -5.98 (95\% CI: - 10.41, 


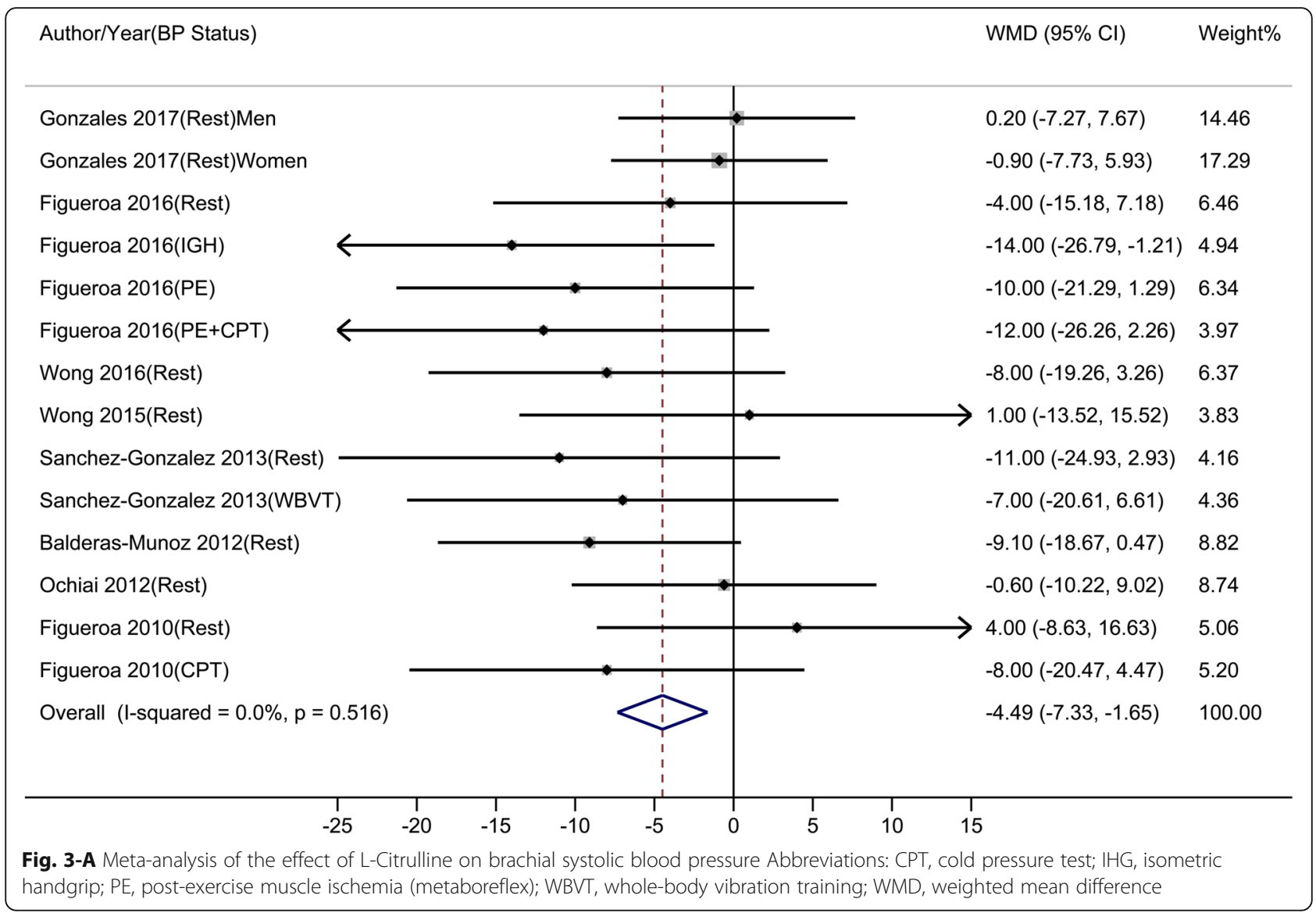

- 1.56) for aortic SBP, and - 4.33 (95\% CI: $-8.69,0.03)$ to -2.60 (95\% CI: $-6.82,1.63)$ for aortic DBP. In summary, the overall estimated WMD of neither brachial BP nor aortic BP were remarkably altered by excluding any single trial (Additional file 13: Figure S13, Additional file 14: Figure S14, Additional file 15: Figure S15, Additional file 16: Figure S16).

\section{Discussion}

Findings from this meta-analysis of 14 trials indicated that L-Cit supplementation had significant effects on lowering brachial BP and aortic SBP, but no effect on aortic DBP. Further analysis according to resting status revealed that L-Cit reduced non-resting brachial and aortic SBP, as well as resting and non-resting brachial DBP, but no effect was found on aortic DBP. Subgroup analyses showed that LCit supplementation reduced brachial SBP in men and those aged < 30 years, and reduced brachial DBP among both genders and age groups.

\section{Results from previous meta-analyses}

There are some differences between our analysis and the previous three meta-analyses. It is notable that in addition to L-Cit, watermelon also contains other bioactive compounds, such as L-Arg and lycopene. Therefore, we treated
L-Cit rather than watermelon as the only supplementation, which differed from the meta-analysis of Mahboobi et al. [17]. Furthermore, Mirenayat et al. [19] analyzed resting BP in five trials and found that L-Cit supplementation had no beneficial effect on either brachial BP or aortic BP. In the present study, we added three more eligible studies $[15,16$, $20]$, and analyzed the effects of L-Cit supplementation on brachial BP and aortic BP according to resting status. Furthermore, meta-regression analyses were carried out to explore potential sources of heterogeneity. Thus, we provided more informative evidence for the effects of L-Cit on BP in this updated meta-analysis.

\section{Results from studies that were not eligible for this meta- analysis}

Some studies were excluded from the present metaanalysis because of unsuitable supplementary methods $[24,25]$, no control group [10, 26], being an acute L-Cit administration [27] or review [28]. Most of these studies illustrated the beneficial effects of L-Cit intervention on BP, except for one [24], which showed no significant effect on resting brachial BP in normotensives. ChurchwardVenne et al. [25] reported that a single dose of $10 \mathrm{~g} \mathrm{~L}$-Cit supplementation co-ingested with whey protein may attenuate $\mathrm{BP}$ responses to exercise but did not reduce 


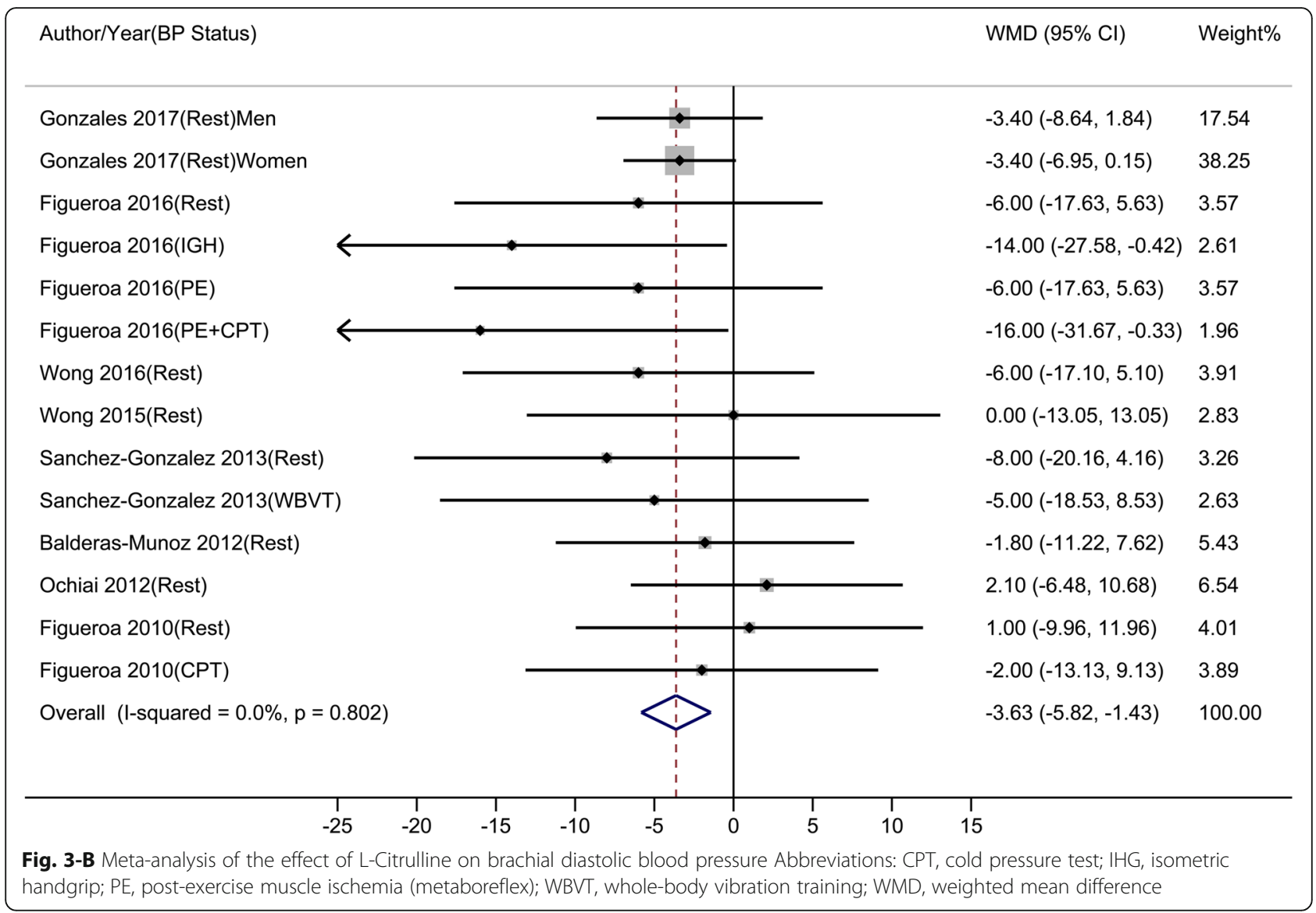

resting $\mathrm{BP}$ in healthy elderly men. Besides exercise $\mathrm{BP}$, Morita et al. [10] found that oral administration of $800 \mathrm{mg}$ of L-Cit for eight weeks resulted in a moderate reduction of resting DBP in adults with vasospastic angina. Orozco-Gutierrez et al. [26] also demonstrated that resting brachial BP was significantly lowered after two months of L-Cit administration. Alsop et al. [27] showed that $3 \mathrm{~g} / \mathrm{d}$ of $\mathrm{L}$-Cit supplementation for one week significantly decreased brachial SBP by $6 \%$ and brachial DBP by $14 \%$ [28]. These preclinical trials supported the hypothesis that L-Cit supplementation may possess the capability to reduce $\mathrm{BP}$.

\section{Findings from animal studies}

Results from animal studies on the effects of L-Cit supplementation on BP control are conflicting. An impaired citrulline-arginine production pathway in the kidneys decreases renal NO, leading to the development of hypertension in spontaneously hypertensive rats (SHRs) [29]. Koeners et al. [29] showed that $2.5 \mathrm{~g} / \mathrm{L}$ of L-Cit supplementation from day seven of gestation to six weeks old of the offspring reduced SBP of both female and male SHRs. Chien et al. [30] showed that $0.25 \% \mathrm{~L}-$ Cit supplementation for eight weeks prevented the transition from prehypertension to hypertension in young hypertensive rats. However, in the study by Mor et al. [31], intravenously administered L-Cit $(10-300 \mu \mathrm{g} / \mathrm{kg} /$ min) did not affect SBP in male rats. Interestingly, Tain et al. found that a $0.25 \%$ L-Cit solution supplemented during the whole period of pregnancy and lactation prevented NG-nitro-L-arginine-methyl ester (NO synthase inhibitor)-induced hypertension in the young offspring rats [32]; but, the intervention raised their BP when they reached adulthood [33].

\section{Potential mechanisms}

L-Cit is an effective exogenous precursor of L-Arg, a natural substrate for NO [34]. As an endotheliumderived relaxing factor, $\mathrm{NO}$ can induce vascular smooth muscle relaxation and vasodilation [35]. Consequently, endothelial-mediated vasodilation leads to BP reduction. In our previous meta-analysis including 11 RCTs, we found that oral L-Arg significantly lowered brachial SBP by $5.39 \mathrm{mmHg}$ and brachial DBP by $2.66 \mathrm{mmHg}$ [13]. The bioavailability of oral L-Cit may be higher than that of oral L-Arg, given the fact that L-Cit bypasses splanchnic extraction and catabolism by the enzyme arginase located in the enterocytes of intestines, liver, and vasculature [36, 37]. 


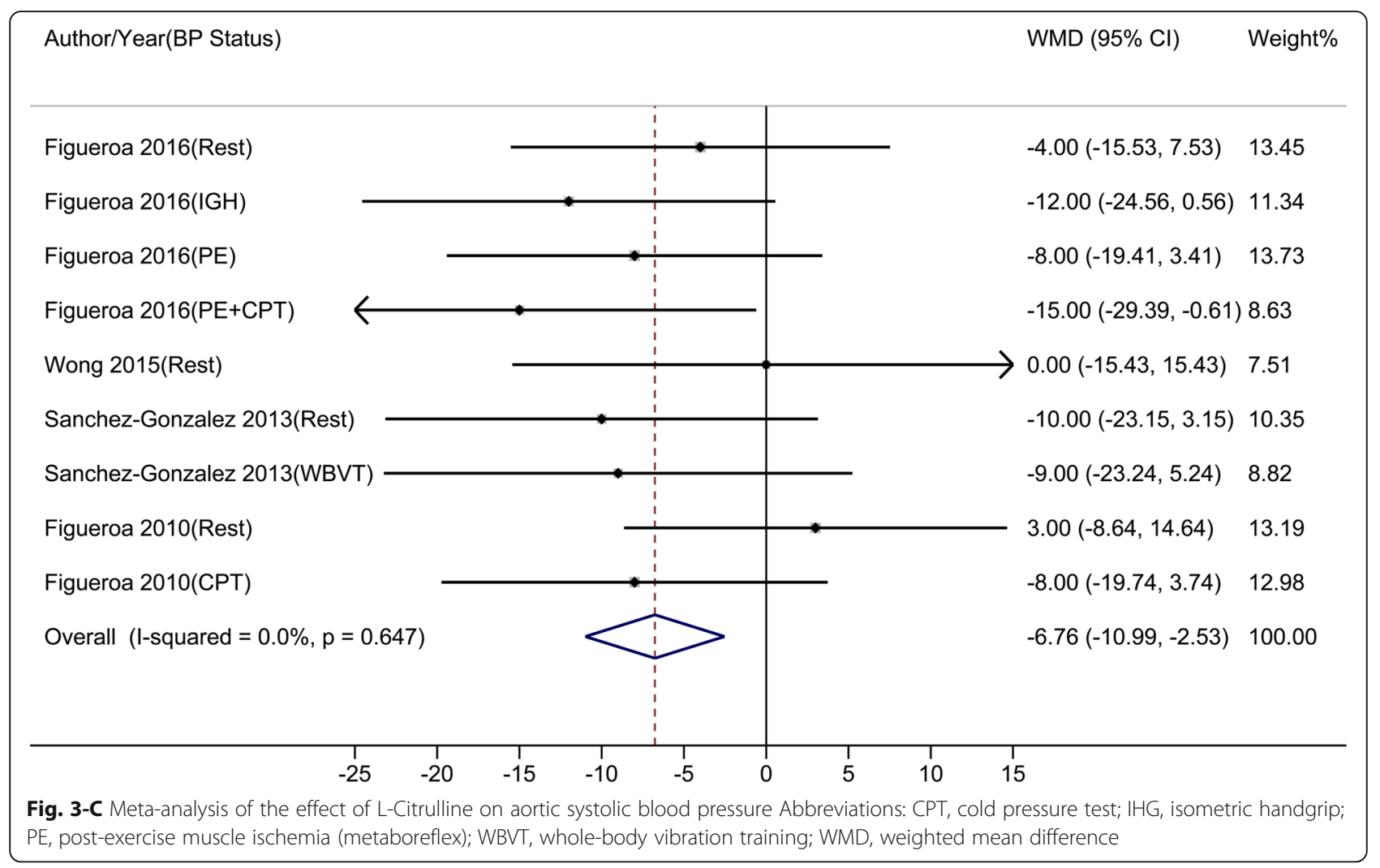

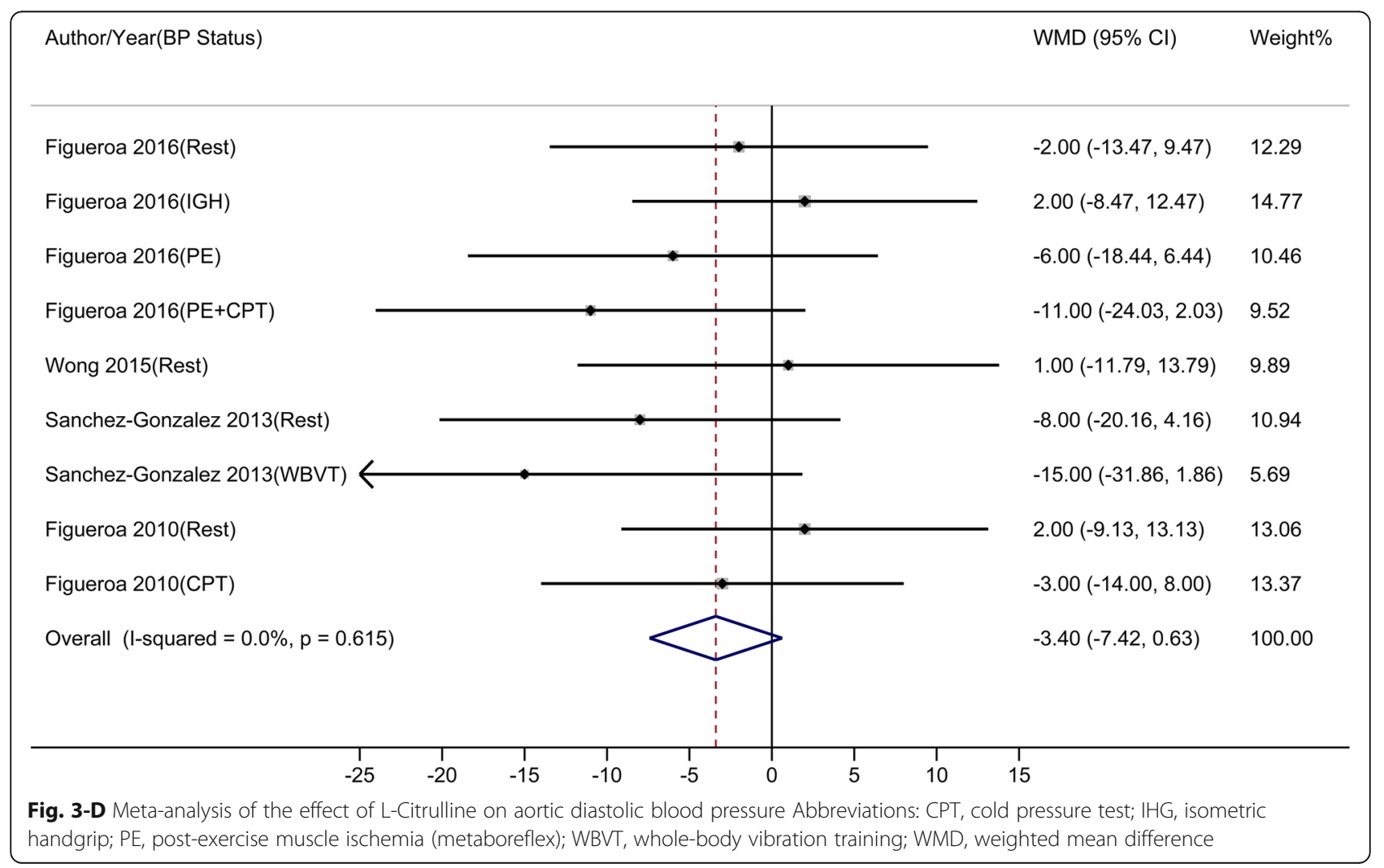




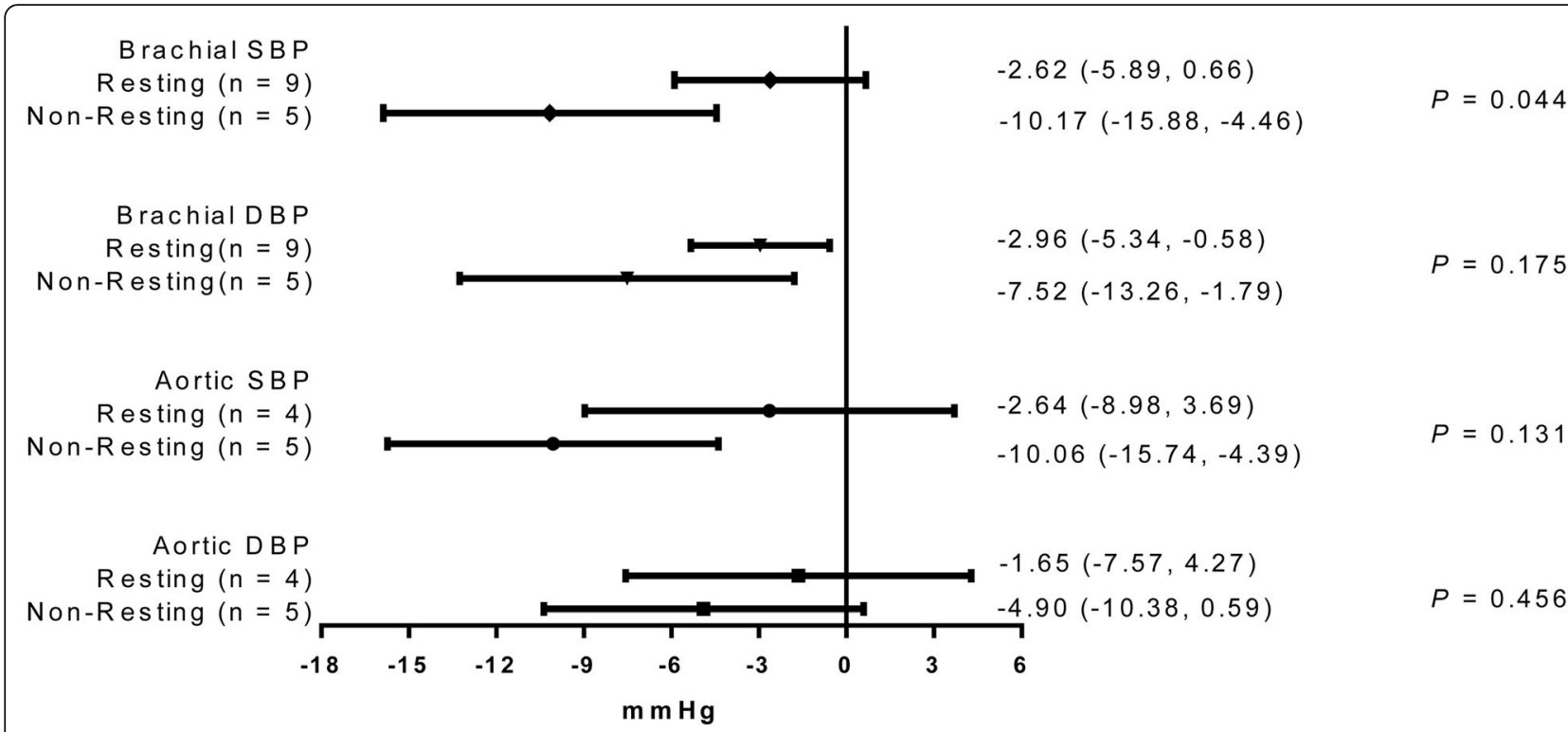

Fig. 4 Analyses of L-citrulline supplementation on brachial and aortic BP according to BP status. Abbreviations: BP, blood pressure; DBP, diastolic blood pressure; P, test for difference between subgroups; SBP, systolic blood pressure

In this regard, several mechanisms underlying the BPlowering effect of L-Cit supplementation have been proposed, including: 1) up-regulation of endothelial-NO synthase (eNOS) [10], a key enzyme for NO production; 2) effective absorption and conversion to L-Arg [38], which is catabolized by eNOS to NO in endothelial cells [11, 12, 39]; 3) inhibition of arginase, a catabolic enzyme that reduces L-Arg bioavailability by converting L-Arg to ornithine and urea [38, 39]; and 4) reduction of asymmetric dimethyl arginine [10], an eNOS competitive inhibitor, to enhance NO production [11, 12, 30,37].

In addition, a stronger effect of oral L-Cit was observed on non-resting BP, including during the post-exercise period, metaboreflex activation, and cold exposure. Elevated $\mathrm{BP}$ response to exercise is probably caused by inadequate perfusion of the active muscles, which is a consequence of a mismatch between supply and demand mediated by increased sympathetic vasoconstriction [9]. In this condition,

Table 2 Sex- and age-stratified analysis of the effect of LCitrulline on brachial systolic and diastolic blood pressure

\begin{tabular}{|c|c|c|c|}
\hline Brachial SBP & & Brachial DBP & \\
\hline WMD (95\% CI) & P & WMD (95\% CI) & $P$ \\
\hline
\end{tabular}

\begin{tabular}{ccccc}
\hline Gender & & & \\
Men & $-4.81(-8.37,-1.24$ & 0.246 & $-4.03(-7.14,-0.91)$ & 0.998 \\
Women & $-2.28(-7.70,3.14)$ & $-3.41(-6.69,-0.14)$ & \\
Age (Years old) & & & \\
$>50$ & $-2.44(-6.13,1.24)$ & 0.113 & $-2.81(-5.35,-0.26)$ & 0.234 \\
$<30$ & $-7.50(-11.97,-3.03)$ & $-6.03(-10.38,-1.68)$ & \\
\hline
\end{tabular}

$B P$ blood pressure; $P$ difference between groups by meta-regression analyses; $W M D$ weighted mean difference
L-Cit supplementation may attenuate exercise BP responses by improvements in regulatory mechanisms of the vascular tone including sympathetic activity and endothelial NO production $[10,12,20]$. On the other hand, $\mathrm{BP}$ response to cold exposure was related to reduced cold-induced systemic vascular reactivity $[16,22]$.

\section{Limitations}

Some limitations of our meta-analysis need to be discussed. First, additional stratified-analysis according to other participant or trial characteristics such as race/ethnicity, health status, dose and duration of intervention were not performed due to the limited number of eligible trials. Dose-response relationship between BP reduction and baseline BP is also yet to be elucidated. Second, the reliability of the results was lowered by the low-quality trials that were included. Nevertheless, sensitivity analyses did not suggest heterogeneity caused by those low-quality trials. Third, potential impacts of publication bias on our findings cannot be excluded, given the tendency to publish larger studies or small studies with encouraging findings.

\section{Conclusion}

L-Cit supplementation significantly decreased nonresting brachial and aortic SBP. Brachial DBP was significantly lowered by L-Cit regardless of resting status. Given the relatively small number of available trials in the stratified analyses and the potential limitations of these trials, the present findings should be interpreted cautiously and need to be confirmed in future welldesigned trials with a larger sample size. 


\section{Supplementary information}

Supplementary information accompanies this paper at https://doi.org/10. 1186/s12986-019-0415-y

Additional file 1: Figure S1. Stratified analysis of the effect of LCitrulline on brachial systolic blood pressure according to BP status. Abbreviations: CPT, cold pressure test; $I H G$, isometric handgrip; PE, postexercise muscle ischemia (metaboreflex); WBVT, whole-body vibration training; WMD, weighted mean difference.

Additional file 2: Figure S2. Meta-regression analysis of brachial systolic blood pressure according to BP status. Abbreviations: CPT, cold pressure test; $I H G$, isometric handgrip; $P E$, post-exercise muscle ischemia (metaboreflex); WBVT, whole-body vibration training; WMD, weighted mean difference.

Additional file 3: Figure S3. Stratified analysis of the effect of LCitrulline on brachial diastolic blood pressure according to BP status. Abbreviations: CPT, cold pressure test; $I H G$, isometric handgrip; PE, postexercise muscle ischemia (metaboreflex); WBVT, whole-body vibration training; WMD, weighted mean difference.

Additional file 4: Figure S4. Meta-regression analysis of brachial diastolic blood pressure according to BP status. Abbreviations: CPT, cold pressure test; $I H G$, isometric handgrip; $P E$, post-exercise muscle ischemia (metaboreflex); WBVT, whole-body vibration training; WMD, weighted mean difference.

Additional file 5: Figure S5. Stratified analysis of the effect of LCitrulline on aortic systolic blood pressure according to BP status. Abbreviations: CPT, cold pressure test; $H_{\mathrm{HG}}$, isometric handgrip; $\mathrm{PE}$, post-exercise muscle ischemia (metaboreflex); WBVT, whole-body vibration training; WMD, weighted mean difference.

Additional file 6: Figure S6. Meta-regression analysis of aortic systolic blood pressure according to BP status. Abbreviations: CPT, cold pressure test; $\mid H G$, isometric handgrip; $P E$, post-exercise muscle ischemia (metaboreflex); WBVT, whole-body vibration training; WMD, weighted mean difference.

Additional file 7: Figure S7. Stratified analysis of the effect of $\mathrm{L}$ Citrulline on aortic diastolic blood pressure according to BP status. Abbreviations: CPT, cold pressure test; $I H G$, isometric handgrip; $P E$, post-exercise muscle ischemia (metaboreflex); WBVT, whole-body vibration training; WMD, weighted mean difference.

Additional file 8: Figure S8. Meta-regression analysis of aortic diastolic blood pressure according to BP status. Abbreviations: CPT, cold pressure test; $I H G$, isometric handgrip; $P E$, post-exercise muscle ischemia (metaboreflex); WBVT, whole-body vibration training; WMD, weighted mean difference.

Additional file 9: Figure S9. Sex-stratified analysis of the effect of LCitrulline on brachial systolic blood pressure. Abbreviations: CPT, cold pressure test; $I H G$, isometric handgrip; $P E$, post-exercise muscle ischemia (metaboreflex); WBVT, whole-body vibration training; WMD, weighted mean difference.

Additional file 10: Figure S10. Age-stratified analysis of the effect of LCitrulline on brachial systolic blood pressure. Abbreviations: CPT, cold pressure test; $I H G$, isometric handgrip; $P E$, post-exercise muscle ischemia (metaboreflex); WBVT, whole-body vibration training; WMD, weighted mean difference.

Additional file 11: Figure S11. Sex-stratified analysis of the effect of LCitrulline on brachial diastolic blood pressure. Abbreviations: CPT, cold pressure test; $I H G$, isometric handgrip; $P E$, post-exercise muscle ischemia (metaboreflex); WBVT, whole-body vibration training; WMD, weighted mean difference.

Additional file 12: Figure S12. Age-stratified analysis of the effect of $L$ Citrulline on brachial diastolic blood pressure. Abbreviations: CPT, cold pressure test; $I H G$, isometric handgrip; PE, post-exercise muscle ischemia (metaboreflex); WBVT, whole-body vibration training; WMD, weighted mean difference.

Additional file 13: Figure S13. Sensitivity analysis of the effect of $L$ Citrulline on brachial systolic blood pressure. Abbreviations: CPT, cold pressure test; $I H G$, isometric handgrip; $P E$, post-exercise muscle ischemia (metaboreflex); WBVT, whole-body vibration training.

Additional file 14: Figure S14. Sensitivity analysis of the effect of LCitrulline on brachial diastolic blood pressure. Abbreviations: CPT, cold pressure test; $I H G$, isometric handgrip; $P E$, post-exercise muscle ischemia (metaboreflex); WBVT, whole-body vibration training.

Additional file 15: Figure S15. Sensitivity analysis of the effect of LCitrulline on aortic systolic blood pressure. Abbreviations: CPT, cold pressure test; $I H G$, isometric handgrip; $P E$, post-exercise muscle ischemia (metaboreflex); WBVT, whole-body vibration training.

Additional file 16: Figure S16. Sensitivity analysis of the effect of LCitrulline on aortic diastolic blood pressure. Abbreviations: CPT, cold pressure test; $I H G$, isometric handgrip; $P E$, post-exercise muscle ischemia (metaboreflex); WBVT, whole-body vibration training.

Additional file 17: Table S1. Summarised search strategies to identify the effects of L-citrulline interventions on blood pressure. (DOCX $15 \mathrm{~kb}$ )

\section{Abbreviations}

BP: Blood pressure; CVD: Cardiovascular disease; DBP: Diastolic blood pressure; eNOS: Endothelial-NO synthase; IGH: Isometric handgrip; L-Arg: LArginine; L-Cit: L-citrulline; NO: Nitric oxide; RCTs: Randomized controlled trials; SBP: Systolic blood pressure; SDs: Standard deviations;

SHRs: Spontaneously hypertensive rats; WBVT: Whole-body vibration training; WMD: Weighted mean difference

\section{Acknowledgements}

Not applicable.

\section{Authors' contributions}

$\mathrm{L}-\mathrm{QQ}$ and L-HC conceived and designed this research; H-HY and X-LL were major contributors in writing the manuscript; W-GZ and A.F. conducted the data collection and analysis. All authors read and approved the final manuscript.

\section{Funding}

This work was supported by the National Key R\&D Program of China [grant numbers 2017YFC1310700, 2017YFC1310701]; the National Natural Science Foundation of China [grant number 81472974]; and the Priority Academic Program Development of Jiangsu Higher Education Institutions (PAPD).

\section{Availability of data and materials}

The datasets used and/or analyzed during the current study are available from the manuscript and the corresponding author on reasonable request.

Ethics approval and consent to participate

Not applicable.

\section{Consent for publication}

Not applicable.

\section{Competing interests}

The authors declare that they have no competing interests.

\section{Author details}

${ }^{1}$ Department of Nutrition and Food Hygiene, School of Public Health Soochow University, Suzhou 215123, China. ${ }^{2}$ Freelancer, Irving, TX 75039 , USA. ${ }^{3}$ Department of Kinesiology and Sport Management, Texas Tech University, Lubbock, TX 79409, USA.

Received: 15 July 2019 Accepted: 12 December 2019 Published online: 26 December 2019

\section{References}

1. Verdecchia P, Angeli F, Reboldi G. Hypertension and atrial fibrillation: doubts and certainties from basic and clinical studies. Circ Res. 2018;122:352-68,

2. Zanchetti A, Thomopoulos C, Parati G. Randomized controlled trials of blood pressure lowering in hypertension: a critical reappraisal. Circ Res. 2015;116:1058-73. 
3. Cooper-DeHoff RM, Johnson JA. Hypertension pharmacogenomics: in search of personalized treatment approaches. Nat Rev Nephrol. 2016;12:110-22.

4. Safar ME, Jankowski P. Central blood pressure and hypertension: role in cardiovascular risk assessment. Clin Sci (Lond). 2009;116:273-82.

5. Williams B, Lacy PS, Thom SM, Cruickshank K, Stanton A, Collier D, et al. Differential impact of blood pressure-lowering drugs on central aortic pressure and clinical outcomes: principal results of the conduit artery function evaluation (CAFE) study. Circulation. 2006;113:1213-25.

6. Delaney EP, Greaney JL, Edwards DG, Rose WC, Fadel PJ, Farquhar WB. Exaggerated sympathetic and pressor responses to handgrip exercise in older hypertensive humans: role of the muscle metaboreflex. Am J Physiol Heart Circ Physiol. 2010;299:H1318-27.

7. Wong A, Alvarez-Alvarado S, Kinsey AW, Figueroa A. Whole-body vibration exercise therapy improves cardiac autonomic function and blood pressure in obese pre- and stage 1 hypertensive postmenopausal women. J Altern Complement Med. 2016;22:970-6.

8. Greaney JL, Kenney WL, Alexander LM. Sympathetic function during whole body cooling is altered in hypertensive adults. J Appl Physiol (1985). 2017; 123:1617-24.

9. Chant B, Bakali M, Hinton T, Burchell AE, Nightingale AK, Paton JFR, et al. Antihypertensive treatment fails to control blood pressure during exercise. Hypertension. 2018;72:102-9.

10. Morita M, Sakurada M, Watanabe F, Yamasaki T, Doi H, Ezaki H, et al. Effects of Oral L-Citrulline supplementation on lipoprotein oxidation and endothelial dysfunction in humans with Vasospastic angina. Immunol Endocr Metab Agents Med Chem. 2013;13:214-20.

11. Ochiai M, Hayashi T, Morita M, Ina K, Maeda M, Watanabe F, et al. Shortterm effects of L-citrulline supplementation on arterial stiffness in middleaged men. Int J Cardiol. 2012;155:257-61.

12. Wong A, Alvarez-Alvarado S, Jaime SJ, Kinsey AW, Spicer MT, Madzima TA, et al. Combined whole-body vibration training and L-citrulline supplementation improves pressure wave reflection in obese postmenopausal women. Appl Physiol Nutr Metab. 2016;41:292-7.

13. Dong JY, Qin LQ, Zhang Z, Zhao Y, Wang J, Arigoni F, et al. Effect of oral Larginine supplementation on blood pressure: a meta-analysis of randomized, double-blind, placebo-controlled trials. Am Heart J. 2011;162:959-65.

14. Figueroa A, Trivino JA, Sanchez-Gonzalez MA, Vicil F. Oral L-Citrulline supplementation attenuates blood pressure response to cold Pressor test in young men. Am J Hypertens. 2010;23:12-6.

15. Balderas-Munãoz K, Castillo-Martínez L, Orea-Tejeda A, Infante-Vázquez O, Utrera-Lagunas M, Martínez-Memije R, et al. Improvement of ventricular function in systolic heart failure patients with oral L-citrulline supplementation. Cardiol J. 2012;19:612-7.

16. Sanchez-Gonzalez MA, Koutnik AP, Ramirez K, Wong A, Figueroa A. The effects of short term L-Citrulline supplementation on wave reflection responses to cold exposure with concurrent isometric exercise. Am J Hypertens. 2013;26:518-26.

17. Mahboobi S, Tsang C, Rezaei S, Jafarnejad S. Effect of L-citrulline supplementation on blood pressure: a systematic review and meta-analysis of randomized controlled trials. J Hum Hypertens. 2019;33:10-21.

18. Barkhidarian B, Khorshidi M, Shab-Bidar S, Hashemi B. Effects of L-citrulline supplementation on blood pressure: a systematic review and meta-analysis. Avicenna J Phytomed. 2019;9:10-20.

19. Mirenayat MS, Moradi S, Mohammadi H, Rouhani MH. Effect of L-Citrulline supplementation on blood pressure: a systematic review and meta-analysis of clinical trials. Curr Hypertens Rep. 2018;20:98.

20. Wong A, Chernykh O, Figueroa A. Chronic I-citrulline supplementation improves cardiac sympathovagal balance in obese postmenopausal women: a preliminary report. Auton Neurosci. 2016;198:50-3.

21. Higgins JPT, Green S (editors). Cochrane Handbook for Systematic Reviews of Interventions 5.1.0. The Cochrane Collaboration. 2011. http://handbook. cochrane.org. Updated March 2011.

22. Figueroa A, Alvarez-Alvarado S, Jaime SJ, Kalfon R. L-Citrulline supplementation attenuates blood pressure, wave reflection and arterial stiffness responses to metaboreflex and cold stress in overweight men. $\mathrm{Br} J$ Nutr. 2016;116:279-85.

23. Gonzales JU, Raymond A, Ashley J, Kim Y. Does I-citrulline supplementation improve exercise blood flow in older adults? Exp Physiol. 2017;102:1661-71.

24. Wax B, Kavazis AN, Weldon K, Sperlak J. Effects of supplemental citrulline malate ingestion during repeated bouts of lower-body exercise in advanced weightlifters. J Strength Cond Res. 2015;29:786-92.
25. Churchward-Venne TA, Cotie LM, MacDonald MJ, Mitchell CJ, Prior T, Baker SK, et al. Citrulline does not enhance blood flow, microvascular circulation, or myofibrillar protein synthesis in elderly men at rest or following exercise. Am J Physiol Endocrinol Metab. 2014;307:E71-83.

26. Orozco-Gutierrez JJ, Castillo-Martinez L, Orea-Tejeda A, Vazquez-Diaz O, Valdespino-Trejo A, Narvaez-David R, et al. Effect of L-arginine or L-citrulline oral supplementation on blood pressure and right ventricular function in heart failure patients with preserved ejection fraction. Cardiol J. 2010;17:612-8.

27. Alsop P, Hauton D. Oral nitrate and citrulline decrease blood pressure and increase vascular conductance in young adults: a potential therapy for heart failure. Eur J Appl Physiol. 2016;116:1651-61.

28. Allerton TD, Proctor DN, Stephens JM, Dugas TR, Spielmann G, Irving BA. ICitrulline Supplementation: Impact on Cardiometabolic Health. Nutrients. 2018;10:921

29. Koeners MP, van Faassen EE, Wesseling S, de Sain-van der Velden M, Koomans HA, Braam B, et al. Maternal supplementation with citrulline increases renal nitric oxide in young spontaneously hypertensive rats and has long-term antihypertensive effects. Hypertension. 2007;50:1077-84.

30. Chien SJ, Lin KM, Kuo HC, Huang CF, Lin YJ, Huang LT, et al. Two different approaches to restore renal nitric oxide and prevent hypertension in young spontaneously hypertensive rats: I-citrulline and nitrate. Transl Res. 2014;163:43-52.

31. Mori A, Morita M, Morishita K, Sakamoto K, Nakahara T, Ishii K. L-Citrulline dilates rat retinal arterioles via nitric oxide- and prostaglandin-dependent pathways in vivo. J Pharmacol Sci. 2015;127:419-23.

32. Tain $Y L$, Lee $C T$, Huang LT. Long-term effects of maternal citrulline supplementation on renal transcriptome prevention of nitric oxide depletion-related programmed hypertension: the impact of gene-nutrient interactions. Int J Mol Sci. 2014;15:23255-68.

33. Tain YL, Hsieh CS, Lin IC, Chen CC, Sheen JM, Huang LT. Effects of maternal $\mathrm{L}$-citrulline supplementation on renal function and blood pressure in offspring exposed to maternal caloric restriction: the impact of nitric oxide pathway. Nitric Oxide. 2010;23:34-41.

34. Bailey SJ, Blackwell JR, Williams E, Vanhatalo A, Wylie LJ, Winyard PG, et al. Two weeks of watermelon juice supplementation improves nitric oxide bioavailability but not endurance exercise performance in humans. Nitric Oxide. 2016;59:10-20.

35. Archer SL, Huang JM, Hampl V, Nelson DP, Shultz PJ, Weir EK. Nitric oxide and CGMP cause vasorelaxation by activation of a charybdotoxin-sensitive $K$ channel by cGMP-dependent protein kinase. Proc Natl Acad Sci U S A. 1994; 91:7583-7.

36. Berkowitz DE, White R, Li D, Minhas KM, Cernetich A, Kim S, et al. Arginase reciprocally regulates nitric oxide synthase activity and contributes to endothelial dysfunction in aging blood vessels. Circulation. 2003;108:2000-6.

37. Papadia C, Osowska S, Cynober L, Forbes A. Citrulline in health and disease. Review on human studies. Clin Nutr. 2018:37:1823-1828.

38. Curis E, Nicolis I, Moinard C, Osowska S, Zerrouk N, Benazeth S, et al. Almost all about citrulline in mammals. Amino Acids. 2005;29:177-205.

39. Figueroa A, Wong A, Jaime SJ, Gonzales JU. Influence of L-citrulline and watermelon supplementation on vascular function and exercise performance. Curr Opin Clin Nutr Metab Care. 2017;20:92-8.

\section{Publisher's Note}

Springer Nature remains neutral with regard to jurisdictional claims in published maps and institutional affiliations.

Ready to submit your research? Choose BMC and benefit from:

- fast, convenient online submission

- thorough peer review by experienced researchers in your field

- rapid publication on acceptance

- support for research data, including large and complex data types

- gold Open Access which fosters wider collaboration and increased citations

- maximum visibility for your research: over $100 \mathrm{M}$ website views per year

At $\mathrm{BMC}$, research is always in progress.

Learn more biomedcentral.com/submission 